\title{
Validation of a Hybrid Grid Scheme of DSMC in Simulating Three-Dimensional Rarefied Gas Flows
}

\author{
Hongli Liu, Jing Fan and Ching Shen \\ Laboratory for High-Temperature Gas Dynamics, Institute of Mechanics, CAS, Beijing 100080, China
}

\begin{abstract}
A hybrid grid scheme is employed in a new version of position element algorithm of the direct simulation Monte Carlo (DSMC) method. The computational domain is divided into uniform rectangular grids to track molecular trajectories efficiently, whereas the body surface is described accurately in a style similar to the unstructured grid technique. This scheme has been applied to hypersonic rarefied gas flows past a sphere and impingement of plume on a flat plate. The calculated results are compared with the experimental data of drag coefficients and density distributions along the stagnation line for the case of sphere, and with distributions of pressure, shear stress and heat flux along the plate surface for the case of plume impingement. Good agreement validates the efficiency and accuracy of the scheme in simulating 3-D rarefied gas flows.
\end{abstract}

\section{INTRODUCTION}

The direct simulation Monte Carlo method [1] is a reliable tool to analyze hypersonic rarefied gas flows. Like most numerical methods, a grid scheme plays an important role in efficient and accurate predictions of DSMC. Bird employed a position element algorithm to calculate the three-dimensional flow fields near a small thruster and around the Shuttle Orbiter [2, 3]. Because the computational domains were divided into uniform rectangular grids, and the body surfaces were approximately represented by the side surfaces of the rectangular grids, the moving molecules were readily indexed to cells. Compared with an algorithm based on an unstructured tetrahedral grid, the position element algorithm is more efficient, but it is sometimes inaccurate to represent the surface details. A new version of position element algorithm [4, 5] was developed that employed a hybrid grid scheme: a computational domain was still divided into a set of rectangular grids, whereas the surface of a body was more accurately described in a style similar to an unstructured grid scheme [6]. Using this hybrid grid scheme, most of simulated molecules are as efficiently tracked as the original position element algorithm, while the small portion in a region adjacent to the body surface are treated delicately like in the unstructured grid scheme.

In this paper, a procedure to mark surface elements is described. A module based on the new marking scheme is incorporated into our general 3-D code of DSMC to simulate hypersonic rarefied gas flows past a sphere and impingement of plume on a flat plate at experimental conditions [7-11]. The calculated results are compared with measured data to validate the efficiency and accuracy of the new position element algorithm in simulating three-dimensional rarefied gas flows. 


\section{A PROCEDURE TO MARK SURFACE ELEMENTS}

Marking surface elements is one of the key steps to implement the new position element algorithm. A procedure used in this paper that can directly and accurately mark and record how a body surface is distributed over the surface elements is summarized as follows:

1) Divide the object body into a number of stations (the space between neighboring stations could be determined according to the actual shape of the object) in the free stream direction, and uniformly distribute the same numbers of points (named station points) on every station contour line with equal angle separations;

2) Divide the object body surface into small triangles (named body surface triangles) by connecting the station points of the two neighboring stations one by one, with no allowance of the overlap of arbitrary two triangles;

3) Divide the whole computational domain into uniform rectangular grids (named position elements);

4) Identify the body surface triangles and the position elements by two series of numbers, and call a body surface triangle related to a position element if one of the following conditions is satisfied:

A. the position element contains at lease one apex of the body surface triangle;

B. when the condition A is not met, at least one side line of the body surface triangle intersects with at least one side surface of the position element;

C. when both condition A and condition B are not met, at least one arris of the position element intersects with the body surface triangle;

5) Identify a position element meeting at least one of the conditions A, B and C in step 4) as a surface element, and record the amount and the serial numbers of the body surface triangles related to the surface element;

6) A deterministic criterion [5] decides the location of a simulated molecule striking the body surface.

\section{RESULTS AND DISCUSSION}

A module based on the procedure above has been incorporated into our general DSMC code [4, 5]. In this code, the variable hard sphere (VHS) model [12] is employed to describe molecular interaction, and the Larsen-Borgnakken model [13] to distribute the translation and internal energies after collisions. A generalized acceptance-rejection technique [14] is used to overcome the singularity in the Larsen-Borgnakke model arising from the vibrational energy. Molecular collision frequency is calculated using the randomly sampled frequency method [15].

\section{Hypersonic rarefied flows around a sphere}

Our first numerical example is a free molecular flow past a sphere. This is easily achieved in the DSMC code by turning off molecular collisions. The theoretical solutions of the incident surface pressure and heat flux may be written as

$$
\frac{2 p_{i}}{\rho_{\infty} u_{\infty}^{2}}=\frac{s \cos \theta \exp \left(-s^{2} \cos ^{2} \theta\right)+\sqrt{\pi}\{1+e r f(s \cos \theta)\}\left(0.5+s^{2} \cos ^{2} \theta\right)}{\sqrt{\pi} s^{2}},
$$


TABLE 1. Free stream conditions in calculating drag coefficients of sphere

\begin{tabular}{ccccc}
\hline Case & $\rho_{\infty}\left(\mathrm{kg} \cdot \mathrm{m}^{-3}\right)$ & $\lambda_{\infty}(\mathrm{m})$ & $\mathrm{Kn}_{\infty}$ & $\mathrm{Re}_{\infty}$ \\
\hline 1 & $1.032 \times 10^{-4}$ & $4.0 \times 10^{-4}$ & $1.0 \times 10^{-2}$ & $1.360 \times 10^{3}$ \\
2 & $1.032 \times 10^{-5}$ & $4.0 \times 10^{-3}$ & $1.0 \times 10^{-1}$ & $1.360 \times 10^{2}$ \\
3 & $1.032 \times 10^{-6}$ & $4.0 \times 10^{-2}$ & $1.0 \times 10^{0}$ & $1.360 \times 10^{1}$ \\
4 & $1.032 \times 10^{-7}$ & $4.0 \times 10^{-1}$ & $1.0 \times 10^{1}$ & $1.360 \times 10^{0}$ \\
5 & $1.032 \times 10^{-8}$ & $4.0 \times 10^{0}$ & $1.0 \times 10^{2}$ & $1.360 \times 10^{-1}$ \\
\hline
\end{tabular}

$$
\frac{2 q_{i}}{\rho_{\infty} u_{\infty}^{3}}=\frac{\left(s^{2}+2\right) \exp \left(-s^{2} \cos ^{2} \theta\right)+\sqrt{\pi} s \cos \theta\left(s^{2}+2.5\right)(1+e r f(s \cos \theta)\}}{\sqrt{\pi} s^{3}},
$$

where $\rho_{\infty}$ and $u_{\infty}$ are the free stream density and velocity, respectively, $s=u_{\infty} / c_{m}$ is the molecular speed ratio, $c_{m}$ is the most probable thermal speed, $\theta$ is the angle between the free stream and the normal direction of surface, and $\operatorname{erf}(\cdots)$ is the error function. Comparison of the DSMC results with the exact solutions shows excellent agreement (Fig.1). This indicates that the hybrid grid scheme is capable of accurately recording the sphere surface.

Legge and Koppenwallner [7] experimentally investigated the Knudsen (Kn) number dependence of drag coefficient for hypersonic rarefied gas flows past a sphere. In experiment, the sphere had a diameter of $0.04 \mathrm{~m}$. The free stream of air was around the Mach (Ma) number of 9.0, and the total temperature $T_{\mathrm{o}}$ was $500 \mathrm{~K}$. The DSMC calculations employs the same flow conditions as experiment, with $\mathrm{Kn}$ based on the sphere diameter varying from 0.01 to 100 (Table 1). Since the experiment was carried out adiabatically, the sphere surface in the simulation is assumed to be diffusely reflecting with the same temperature as $T_{0}$. The DSMC drag coefficients agree well with the measured data [7] over the entire flow regime, and approach to the theoretical value, 2.685 , in the free molecular regime.

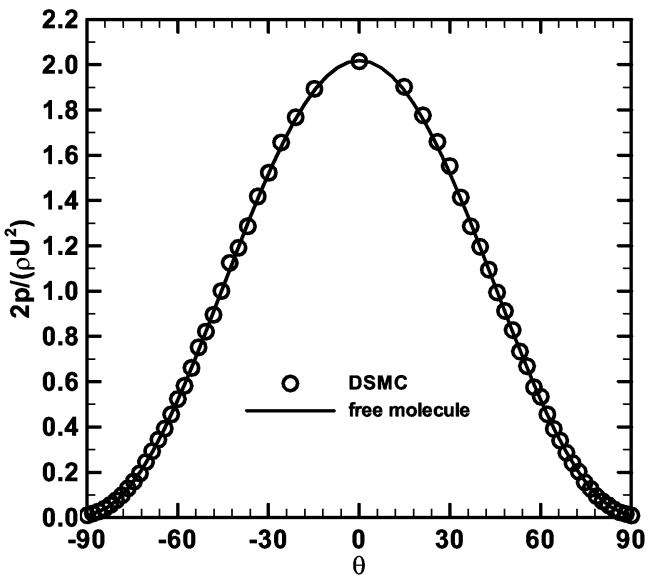

(a) Pressure

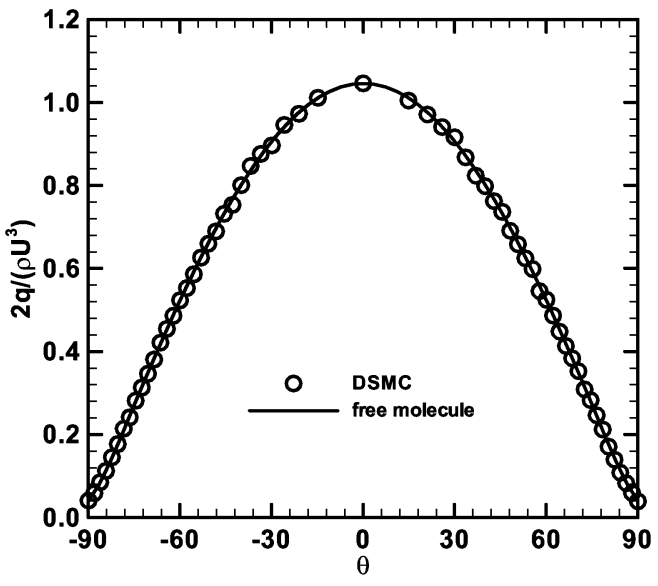

(b) Heat flux

FIGURE 1. Comparison of DSMC and theoretical pressure and heat flux profiles along a sphere surface in free molecular limit. 


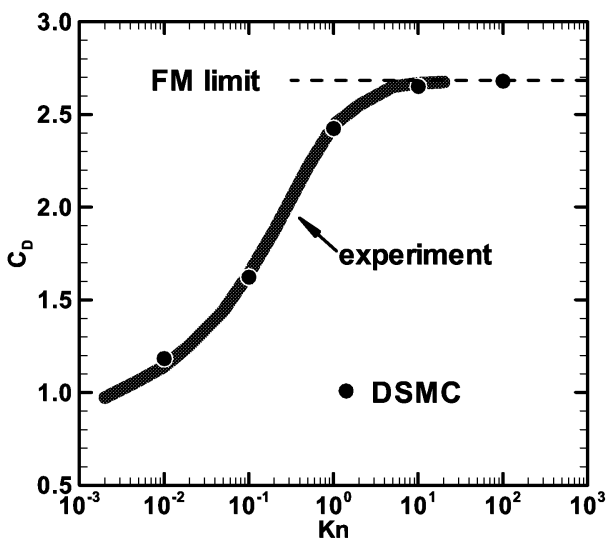

FIGURE 2. Drag coefficients of hypersonic rarefied gas flows past a sphere given by the DSMC method and experiment [7].

Russell [8] measured density distributions along the stagnation line of a sphere. In experiment, the Mach number of nitrogen was 4.2 or 4.38 . The total temperature was $300 \mathrm{~K}$, so was the surface temperature. The DSMC simulation is carried out at experimental conditions with different Reynolds numbers. The calculated results are compared with Russell's data in Fig.3, where the normalized factors are the sphere radius and free stream density, respectively. As can be seen, agreement is remarkable.

\section{Impingement of plume on a flat plate}

As shown in Fig.4, in experiment carried out by Legge [9, 10] and Doring [11], respectively, a plume of molecular nitrogen exited from a sonic orifice expanded through a vacuum chamber, and impinged onto a flat plate.

The distance between the orifice and the plate distance, $L$, was $40 \mathrm{~mm}$, and the orifice diameter, $\mathrm{r}^{*}$, was $1 \mathrm{~mm}$. The stagnation pressure and temperature were $1000 \mathrm{~Pa}$ and $300 \mathrm{~K}$, respectively. The plate surface temperature was the same as the total temperature of the gas. The background pressure, $p_{b}$, was maintained at $0.045 \mathrm{~Pa}$ in measurements of pressure and shear stress distributions over the plate [9, 10], and at $0.090 \mathrm{~Pa}$ in those of heat flux distributions [11]. In a previous investigation of this issue using the DSMC method, the exit conditions were derived based on an assumption that the flow was sonic at the exit [16]. The same assumption is employed here, and the velocity, temperature, and number density at exit are $323 \mathrm{~m} / \mathrm{s}, 252 \mathrm{~K}, 1.53 \times 10^{-23} \mathrm{~m}^{-3}$, respectively.

TABLE 2. Free stream conditions in calculating density distributions along the stagnation line of sphere

\begin{tabular}{cccc}
\hline Case & 1 & 2 & 3 \\
\hline $\mathrm{Re}_{\infty}$ & 30 & 50 & 100 \\
$M a_{\infty}$ & 4.2 & 4.38 & 4.38 \\
$R(\mathrm{~m})$ & $6.4 \times 10^{-3}$ & $6.4 \times 10^{-3}$ & $1.27 \times 10^{-2}$ \\
$\lambda(\mathrm{m})$ & $1.325 \times 10^{-3}$ & $8.320 \times 10^{-4}$ & $8.320 \times 10^{-4}$ \\
\hline
\end{tabular}




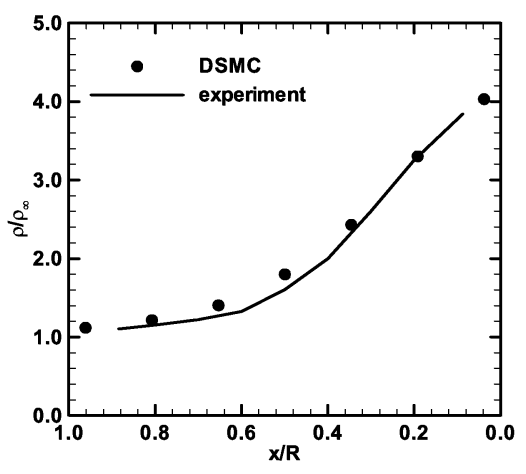

(a) $\mathrm{Re}=30$

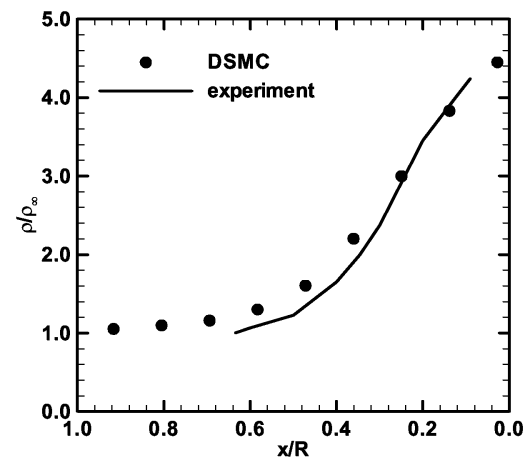

(b) $\mathrm{Re}=50$

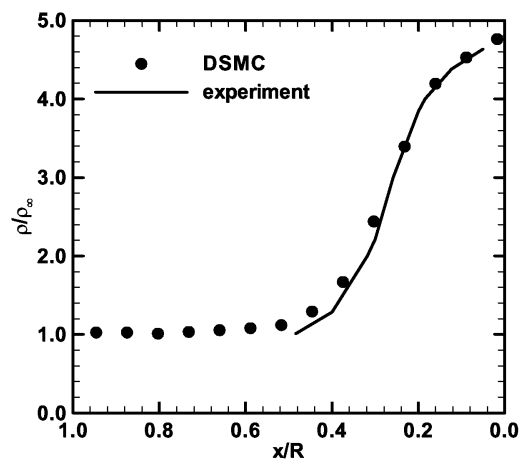

(c) $\operatorname{Re}=100$

FIGURE 3. Comparison of DSMC density distributions along the stagnation line of a sphere with measured data [8].

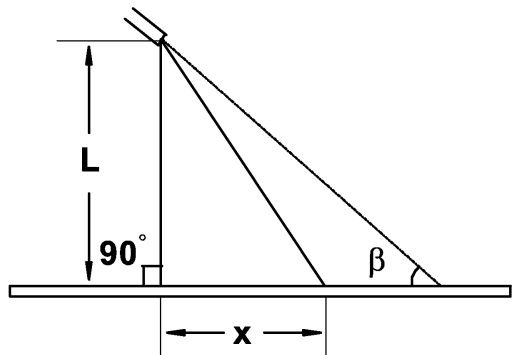

FIGURE 4. Schematic diagram of impingement of plume on a flat plate 

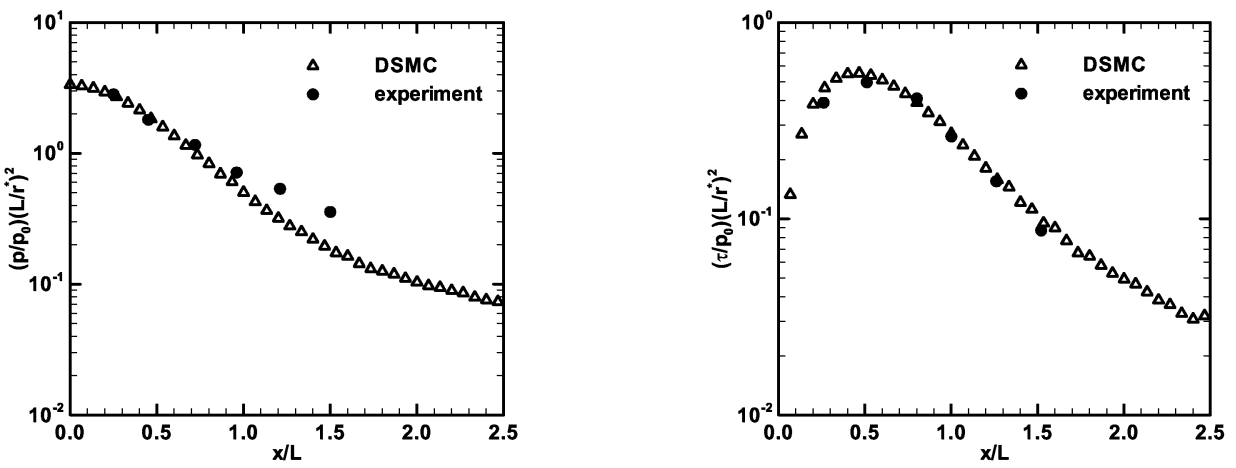

(a) $\beta=90^{\circ}$
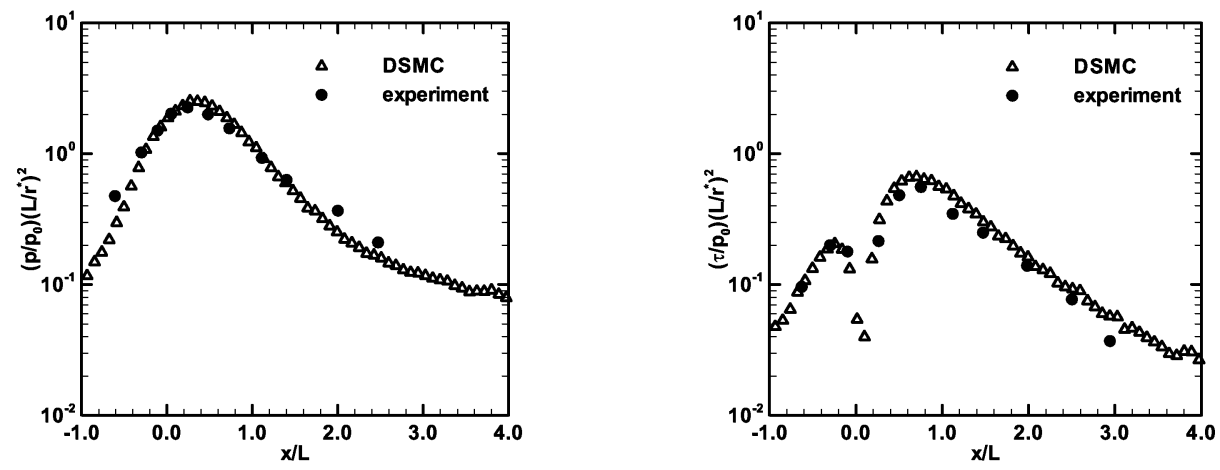

(b) $\beta=45^{\circ}$
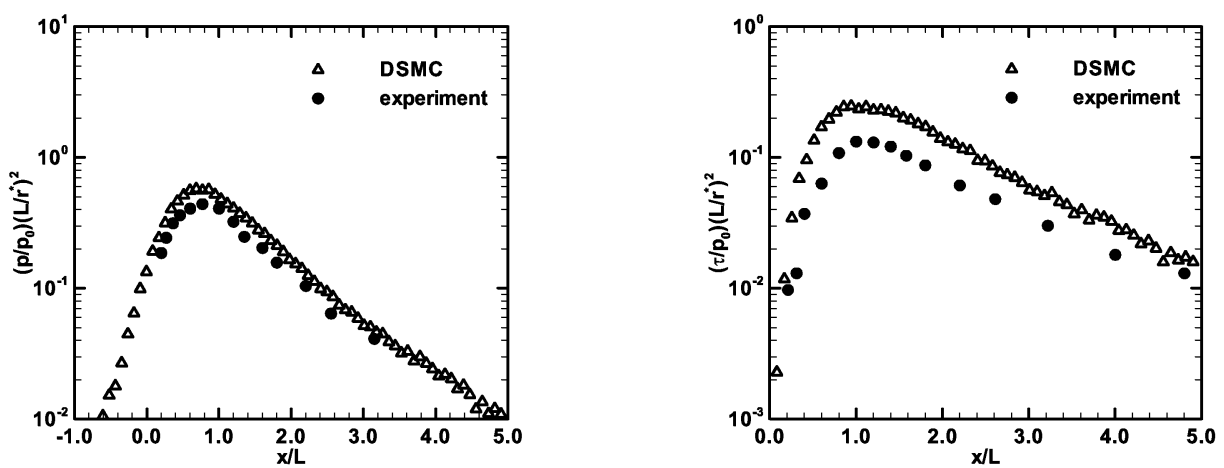

(c) $\beta=0^{\circ}$

FIGURE 5. Comparison of surface pressure and shear stress distributions in an impingement of plume on a flat plate given by DSMC with experimental data $[9,10]$. Pressure: left; shear stress: right. 


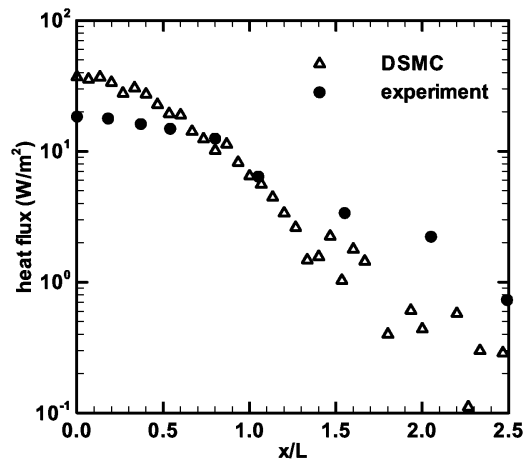

(a) $\beta=90^{\circ}$

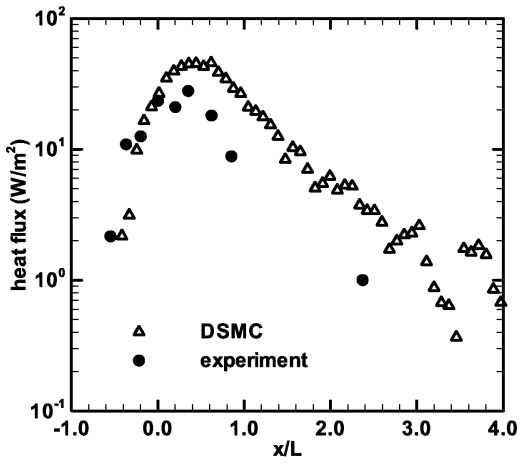

(b) $\beta=45^{\circ}$

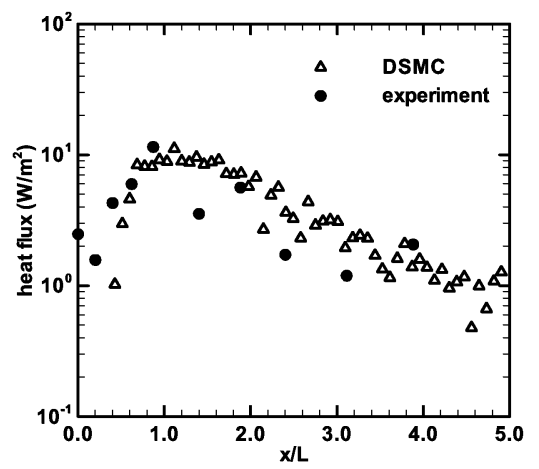

(c) $\beta=0^{\circ}$

FIGURE 6. Comparison of surface heat flux distributions in an impingement of plume on a flat plate given by DSMC with experimental data [11].

Figures 5-6 compare the pressure, shear stress and heat flux distributions along the flat plate given by the DSMC method and experiment [9-11], respectively. Good agreements are obtained for all the distributions of pressure, and those of the shear stress at $\beta=90^{\circ}$ and $\beta=45^{\circ}$, whereas there was a small difference between the calculated and measured results of the shear stress at $\beta=0^{\circ}$. The heat fluxes, though obviously scattered in both the calculations and experiments, generally agree with each other in trend.

\section{ACKNOWLEDGEMENT}

The work was supported by NNSFC under Grant 19889209. 


\section{REFERENCES}

1. Bird, G. A., Molecular Gas Dynamics and the Direct Simulation of Gas Flows. Clarendon Press, Oxford, 1994.

2. Bird, G. A., Application of the DSMC method to the full shuttle geometry, AIAA paper 90-1692, 1990.

3. Bird, G. A., Influence of local configuration on the backflow from small rocket thrusters, AIAA Paper 90-0147, 1990.

4. Shen, C., Fan, J., Hu, Z. H., Xu, X. Y., “A new version of position element algorithm of DSMC calculation of three-dimensional transitional flows" in Rarefied Gas Dynamics, edited by C. Shen, 1997, pp. 162-167.

5. Fan, J., Liu, H. L., Shen, C., Chen, L. M., Acta Aerodynamics Sinica (in Chinese) 18, 180-187 (2000).

6. Dietrich, S., Boyd, I. D., Journal of Computational Physics 126, 328-342 (1996).

7. Legge, H., Koppenwallner, G., "Sphere drag measurements in a free jet and a hypersonic low density tunnel," in Rarefied Gas Dynamics, edited by D. Dini, 1970, pp. 481-488

8. Russell, D. A., Physics of Fluids 8, 1679-1685 (1968)

9. Legge, H., "Shear stress and pressure in plume impingement flow" in Rarefied Gas Dynamics, edited by V. Boffi and C. Cercignani, 1986, pp. 523-538.

10. Legge, H., "Plume Impingement Forces on Inclined Flat Plates" in Rarefied Gas Dynamics, edited by A.E. Beylich, 1991 , pp. $955-962$

11. Doring, S., “Experimental Plume Impingement Heat Transfer on Inclined Flat Plates,” DLR, TRIB $222-90$ A $36,1990$.

12. Bird, G. A., Progr. Astro. Aero. 74, 239-255 (1981)

13. Borgnakke, C., Larsen, P. S., J. Comput. Phys. 18, 405-420 (1975)

14. Shen, C., Wu, W. Q., Hu, Z. H., and Xu, X. Y., Acta Aerodynamics Sinica (in Chinese) 9, 1-7 (1991)

15. Fan, J., and Shen, C., "A New Algorithm of the Direct Simulation Monte-Carlo Method-the Randomly Sampled Frequency Method” in Methods, Theories, and Applications of CFD, Science Press, Beijing, 1992, pp.127-130.

16. Kannenberg, K. C., Boyd, I. D., J.ournal of Thermophysics and Heat Transfer 13, 226-235 (1999) 